\title{
Câncer de Mama Multifocal: Relato de Caso
}

doi: https://doi.org/10.32635/2176-9745.RBC.2020v66n4.956

\author{
Multifocal Breast Cancer: Case Report \\ Cáncer de Mama Multifocal: Relato de caso
}

\author{
Gabriela Chielli'; João Ramalho Borges²; Gabriel Lucas Martins³; Thiago Fernandes de Lacerda4; Welington Lombardi5; Luciana \\ Borges Lombardí
}

\begin{abstract}
RESUMO
Introduçáo: O câncer de mama é a neoplasia que mais acomete o sexo feminino, sendo a primeira causa de morte por câncer em mulheres. O carcinoma mamário representa um grupo heterogêneo de doenças. Casos individuais diferem uns dos outros na morfologia, fenótipo e prognóstico. As patologias malignas das mamas podem se manifestar como tumores unifocais, multifocais e/ou multicêntricos. A incidência de tumores multifocais e multicêntricos no câncer de mama varia de 13\% a 70\%. Relato do caso: Paciente L.C., sexo feminino, 65 anos, com relato de nódulo palpável em mama direita em setembro de 2015. O estudo anatomopatológico do nódulo mostrou carcinoma intraductal. Realizada quadrantectomia, com anatomopatológico que identificou carcinoma papilífero bem diferenciado intracístico e invasivo da mama, associado a componente intraductal cribriforme e papilar, com margens e linfonodo sentinela livres e imuno-histoquímica compatível com perfil triplo-negativo. Em fevereiro de 2019, apresentou duas novas lesôes em mama contralateral, identificadas como carcinoma ductal invasivo multifocal com papiloma intraductal associado, e carcinoma ductal invasivo associado a componente intraductal in situ dos tipos papilar, sólido e cribriforme, com imuno-histoquímica com perfis moleculares distintos entre si, sendo uma lesão do tipo luminal A e a outra, luminal híbrido. Conclusáo: Este estudo relata um caso de uma paciente que apresentou lesôes neoplásicas em ambas as mamas, em tempos distintos e com perfis histológicos e imuno-histoquímicos diferentes. Dessa forma, destacam-se a raridade do caso e a relevância da terapia dirigida a alvos específicos, uma vez que a paciente apresentava lesôes com perfis moleculares distintos. Palavras-chave: Neoplasias da Mama; Carcinoma Ductal de Mama; Mastectomia Segmentar; Quimioterapia Adjuvante; Radioterapia Adjuvante.
\end{abstract}

\begin{abstract}
Introduction: Breast cancer is the neoplasm that most affects females, being the first cause of death by cancer in women. Breast carcinoma is a heterogeneous group of diseases. Individual cases differ from each other in morphology, phenotype and prognosis. Malignant breast pathologies can manifest as single, multifocal and/or multicentric tumors. The incidence of multifocal and multicentric tumors in breast cancer varies from $13 \%$ to $70 \%$. Case report: Patient L.C., female, 65 years old, with a palpable nodule in the right breast in September 2015. The anatomopathological study of the nodule showed intraductal carcinoma. She underwent quadrantectomy, with anatomopathological examination that identified well-differentiated intracystic and invasive papillary carcinoma of the breast, associated with a cribriform and papillary intraductal component, with free sentinel lymph node and margins and immunohistochemistry compatible with triple negative profile. In February 2019, she presented two new lesions in contralateral breast, identified as invasive multifocal ductal carcinoma, with associated intraductal papilloma, and invasive ductal carcinoma, associated with an in situ intraductal component of the papillary, solid and cribriform types, with immunohistochemistry with different molecular profiles, being one lesion classified as luminal A and the other, hybrid luminal. Conclusion: This study reports a case of a patient who had neoplastic lesions in both breasts, at different times and with distinctive histological and immunohistochemical profiles. Thus, the rarity of the case and the relevance of the therapy aimed at specific targets are highlighted, since the patient presented lesions with different molecular profiles.

Key words: Breast Neoplasms; Carcinoma, Ductal, Breast; Mastectomy, Segmental; Chemotherapy, Adjuvant; Radiotherapy, Adjuvant.
\end{abstract}

\section{RESUMEN}

Introducción: El cáncer de mama es la neoplasia que más afecta a las mujeres, siendo la primera causa de muerte por cáncer en las mujeres. El carcinoma de mama representa un grupo heterogéneo de enfermedades. Los casos individuales difieren entre sí en morfología, fenotipo y pronóstico. Las patologías mamarias malignas pueden manifestarse como tumores únicos, multifocales y/o multicéntricos. La incidencia de tumores multifocales y multicéntricos en el cáncer de mama varía del 13\% al 70\%. Relato del caso: Paciente L.C., mujer, 65 años, con un nódulo palpable en el seno derecho en septiembre de 2015. El estudio anatomopatológico de la lesión mostró carcinoma intraductal. La paciente se sometió a una cuadrantectomía, con un examen anatomopatológico que identificó un carcinoma papilar invasivo e intraquístico bien diferenciado de mama, asociado con un componente intraductal cribiforme y papilar, con ganglio linfático y márgens libres y inmunohistoquímica compatible con perfil triple negativo. En febrero de 2019, presentó dos nuevas lesiones en el seno contralateral, identificadas como carcinoma ductal multifocal invasivo, con papiloma intraductal asociado y carcinoma ductal invasivo, asociado con un componente intraductal in situ de los tipos papilar, sólido y cribiforme, con inmunohistoquímica con diferentes perfiles moleculares, siendo una lesión del tipo luminal A y la otra, luminal híbrida. Conclusión: Este estudio reporta un caso de una paciente que tenía lesiones neoplásicas en ambos senos, en diferentes momentos y con diferentes perfiles histológicos e inmunohistoquímicos. Por lo tanto, se destaca la rareza del caso y la relevancia de la terapia dirigida a objetivos específicos, una vez que la paciente presentó lesiones con diferentes perfiles moleculares.

Palabras clave: Neoplasias de la Mama; Carcinoma Ductal de Mama; Mastectomía Segmentaria; Quimioterapia Adyuvant; Radioterapia Ayuvante.

\footnotetext{
'Hospital Santa Casa de Misericórdia de Ribeirão Preto. Ribeirão Preto (SP), Brasil. Orcid iD: https://orcid.org/0000-0002-1443-9589

${ }^{2}$ Universidade de Araraquara (Uniara). Araraquara (SP), Brasil. Orcid iD: https://orcid.org/0000-0001-6255-0700

${ }^{3}$ Uniara. Araraquara (SP), Brasil. Araraquara (SP), Brasil. Orcid iD: https://orcid.org/0000-0002-0292-0973

${ }^{4}$ Uniara. Araraquara (SP), Brasil. Araraquara (SP), Brasil. Orcid iD: https://orcid.org/0000-0002-2632-0278

${ }^{5}$ Uniara. Araraquara (SP), Brasil. Orcid iD: https://orcid.org/0000-0001-6909-4744

${ }^{6}$ Uniara. Araraquara (SP), Brasil. Orcid iD: https://orcid.org/0000-0001-8278-4379

Endereço para correspondência: Gabriela Chielli. Avenida Armando Corrêa de Siqueira, 1273 - Vila Harmonia. Araraquara (SP), Brasil. CEP $14802-580$. E-mail: gabi_chielli@hotmail.com
} 


\section{INTRODUÇÃO}

O câncer de mama é considerado um problema de saúde pública, sendo a neoplasia maligna mais incidente em mulheres em nível mundial ${ }^{1}$. No Brasil, espera-se que ocorram 66.280 novos casos de câncer de mama para cada ano do triênio 2020-2022, valor que corresponde a um risco estimado de 61,61 casos novos a cada $100 \mathrm{mil}$ mulheres $^{2}$. No ano de 2018, ocorreram 17.763 mortes no país em decorrência de câncer de mama, sendo 17.572 no sexo feminino ${ }^{3}$.

O carcinoma mamário representa um grupo heterogêneo de doenças. Os casos individuais diferem uns dos outros na morfologia, fenótipo e prognóstico. Por meio de técnicas de microarranjos de DNA e análises, cinco grupos genéticos distintos da doença foram descritos: luminal A, luminal B, human epidermal growth factor receptor 2 (HER-2) positivo, basal-símile e tumores normais de mama. Esses subtipos tumorais podem ser também identificados com acurácia suficiente durante a rotina diagnóstica, por meio de painel de marcadores imuno-histoquímicos, incluindo anticorpos para rastreio de receptores de estrogênio (ER), receptores de progesterona $(\mathrm{PR})$, superexpressão da oncoproteína c-erbB-2 (HER-2) e alguns marcadores mioepiteliais ${ }^{4}$.

As patologias malignas das mamas podem se manifestar como tumores unifocais, multifocais (MF) e/ ou multicêntricos $(\mathrm{MC})$. A incidência de tumores $\mathrm{MF}$ e MC no câncer de mama varia de $13 \%$ a $70 \% 5$. Tumores de mama MF e MC foram associados a fatores prognósticos adversos, como estadiamento clínico avançado, maior tamanho do tumor e invasão linfovascular. Há uma correlação significativa entre cânceres de mama MF e MC, com uma taxa aumentada de metástase linfonodal axilar e maior estadiamento N. Pacientes com tumores de mama MF e MC têm menor sobrevida em cinco anos e menor sobrevida global, quando comparados com pacientes com cânceres de mama unifocais ${ }^{6}$.

Para propósitos práticos, a distinção entre tumores MF e MC é baseada na topografia e critérios histológicos. Tumores MF são definidos quando um único quadrante é envolvido, e tumores MC quando dois ou mais quadrantes são envolvidos. Alguns autores distinguem tumores $\mathrm{MF}$ de tumores $\mathrm{MC}$ com base na suposição de que cânceres de mama MF surgem dentro do mesmo sistema de ductos e cânceres de mama MC, de sistemas de ductos diferentes ${ }^{6}$. Não existe uma definição radiológica, mas, geralmente, tumores MF são considerados quando há uma distância menor ou igual a $5 \mathrm{~cm}$ entre as lesóes, e tumores $\mathrm{MC}$, quando a distância é maior que $5 \mathrm{~cm}$ entre as lesôes ${ }^{6}$.

A detecção de câncer de mama MF e MC aumentou em razão dos avanços nas técnicas de imagem nos últimos anos. Apesar da prevalência crescente, as implicaçóes prognósticas e de manejo do carcinoma MF de mama são incertas ${ }^{7}$. A maioria das diretrizes recomenda a avaliação de marcadores biológicos apenas no maior foco tumoral. Essa abordagem pode levar a uma subestimação do subtipo molecular e subtratamento de pacientes ${ }^{8}$.

Em janeiro de 2015, foi publicado no New England Journal of Medicine um estudo que avaliou a associação de paclitaxel e trastuzumabe adjuvante em pacientes com lesões de até $3 \mathrm{~cm}$ e linfonodos negativos. Até então, não havia estudos que abordassem esse grupo de pacientes, pois a maioria não era elegível para os principais ensaios com trastuzumabe adjuvante. Tal estudo mostrou benefício para esse perfil de paciente, em termos de sobrevida?

Em se tratando de hormonioterapia em pacientes pós-menopausa, uma metanálise realizada pelo Early Breast Cancer Trialists' Collaborative Group (EBCTCG) ${ }^{10}$ avaliou 31.920 mulheres e comparou o uso de tamoxifeno com inibidores da aromatase, concluindo que o uso de inibidores da aromatase por cinco anos seria superior ao uso de tamoxifeno por cinco anos. O estudo concluiu ainda que o uso de tamoxifeno por dois a três anos, seguido de inibidor de aromatase por dois a três anos, ou uso de inibidor de aromatase por dois a três anos, são relativamente semelhantes, sendo ambos superiores ao uso de tamoxifeno por cinco anos ${ }^{10}$.

O objetivo deste trabalho é relatar o caso de uma paciente que apresentou lesôes neoplásicas em ambas as mamas, em tempos distintos e com perfis histológicos e imuno-histoquímicos diferentes, destacando-se a raridade do caso e a relevância de terapias dirigidas a alvos específicos, uma vez que a paciente apresentou lesões com perfis moleculares distintos.

O presente estudo foi aprovado pelo Comitê de Ética em Pesquisa (CEP) da Universidade de Araraquara, sob o no. CAAE: 32059020.8.0000.5383. A autorização para publicação foi fornecida pela própria paciente, por meio de assinatura do Termo de Consentimento Livre e Esclarecido (TCLE).

\section{RELATO DO CASO}

L.C., sexo feminino, 65 anos, menarca aos 12 anos, G2P2A0, hipertensa, sem outras patologias, história pessoal ou familiar pregressa de câncer, menopausada, com relato de nódulo palpável em mama direita em setembro de 2015. Ao exame físico, apresentou nódulo de cerca de $3 \mathrm{~cm}$ em quadrante superior medial direito, endurecido e aderido aos planos profundos. Possuía mamografia classificada como Breast Imaging-Reporting and Data System (BI-RADS) 0 e punção-biópsia aspirativa por agulha fina (PBAAF) que evidenciou atipias celulares, 
tendo sido encaminhada ao Serviço de Mastologia, no Ambulatório de Saúde da Mulher, em Araraquara, SP.

Realizou-se core biopsy do nódulo, a qual mostrou carcinoma intraductal. Paciente foi então submetida à quadrantectomia direita, com biópsia de linfonodo sentinela. O anatomopatológico da lesão identificou carcinoma papilífero bem diferenciado intracístico e invasivo da mama, associado a componente intraductal cribriforme e papilar, com margens e linfonodo sentinela livres, medindo $2,7 \times 2,0 \times$ $1,8 \mathrm{~cm}$ e distante da pele $1,9 \mathrm{~cm}$, estádio pT2 pN0. O perfil imuno-histoquímico mostrou-se negativo para RE, RP e HER-2; e positividade de mais de $90 \%$ para Ki-67, sendo então classificado como padráo molecular subtipo basal-símile. Nos exames de estadiamento, não havia evidências de doença a distância. A paciente foi encaminhada para o Serviço de Oncologia da Santa Casa de Misericórdia de Araraquara, SP, para realização de tratamento adjuvante.

Após realizar seis ciclos de quimioterapia com esquema FAC adjuvante (doxorrubicina $50 \mathrm{mg} / \mathrm{m}^{2}$, associada à ciclofosfamida $500 \mathrm{mg} / \mathrm{m}^{2}$ e 5 -fluoracil $500 \mathrm{mg} / \mathrm{m}^{2}$ ), e radioterapia adjuvante na dose de 5.040 cGy em 28 FR mais boost com 1.000 cGy em 5 FR, retorna ao ambulatório de mastologia para seguimento ginecológico pós-tratamento. Em fevereiro de 2019, apresentou mamografia BI-RADS 0 e ultrassonografia de mamas BI-RADS 4. Foi identificado novo nódulo, oval, lobulado, maior eixo paralelo à pele, com $0,9 \mathrm{x}$ $0,7 \mathrm{~cm}$, localizado na transiçấo do quadrante superior medial com a regiáo retroareolar da mama esquerda, com $2 \mathrm{~cm}$ de profundidade. Foi solicitada core biopsy, que evidenciou carcinoma mamário invasivo T1N0MX, sendo posteriormente realizada quadrantectomia com biópsia de linfonodo sentinela.

Durante o ato cirúrgico, foram identificadas duas lesões. $\mathrm{Na}$ avaliação anatomopatológica, a lesão maior, localizada em quadrante central, medindo $1,8 \times 1,5 \mathrm{x}$ $1,3 \mathrm{~cm}$, foi classificada como carcinoma ductal invasivo MF, com papiloma intraductal associado, com margens cirúrgicas livres de comprometimento neoplásico. A lesão menor, localizada na união dos quadrantes laterais, medindo $1,1 \times 1,0 \times 0,8 \mathrm{~cm}$, foi classificada como carcinoma ductal invasivo, associado a componente intraductal in situ do tipo papilar, sólido e cribriforme; com margens cirúrgicas e linfonodo sentinela livres. Paciente em estádio mpT1c mpN0.

A imuno-histoquímica, em relação à lesão menor, se mostrou com o seguinte perfil: RE+ 90\%, RP+ 80\%, HER-2-, P53+ 50\%, Ki-67+ em menos de 5\%, com padrão molecular subtipo luminal $\mathrm{A}$. Em relação ao tumor maior, o perfil imuno-histoquímico foi RE+ $60 \%, \mathrm{RP}+80 \%$, HER-2+++, P53-, Ki-67+ 5\%, com padrão molecular subtipo luminal híbrido. A paciente foi então novamente encaminhada ao Serviço de Oncologia da Santa Casa de Misericórdia de Araraquara, SP, para tratamento adjuvante com radioterapia, quimioterapia e hormonioterapia.

Realizada quimioterapia com esquema $\mathrm{TH}$, o qual consiste em paclitaxel em uma dose semanal de $80 \mathrm{mg} / \mathrm{m}^{2}$ associado trastuzumabe durante 12 semanas, na dose de ataque de $8 \mathrm{mg} / \mathrm{kg}$, seguido de nove meses de trastuzumabe em monoterapia, na dose de manutençáo de $6 \mathrm{mg} / \mathrm{kg}$. Optou-se, também, pela realização de hormonioterapia com tamoxifeno e radioterapia com 60 Gy em 30 FR. Atualmente, a paciente se encontra ainda em uso de trastuzumabe e tamoxifeno.

Paciente em programação de realização de cinco anos de hormonioterapia. Com base na metanálise do EBCTCG, quando em uso por dois anos de tamoxifeno, o paciente realizará switch para inibidor de aromatase, até completar cinco anos de hormonioterapia.

\section{DISCUSSÃO}

O câncer de mama é uma doença comum e pode ser classificada como problema de saúde pública em decorrência de sua alta incidência. $\mathrm{O}$ carcinoma papilífero intracístico corresponde a $0,5 \%-1 \%$ de todos os carcinomas de mama, sendo considerado um tumor maligno raro $^{11,12}$. Lesão esta que a paciente apresentou inicialmente.

Existe uma grande variedade de tipos histológicos e moleculares de carcinomas de mama in situ e invasor. O tipo histológico invasor mais comum é o carcinoma ductal infiltrante não especificado, o qual representa de $70 \%$ a $80 \%$ de todos os tumores de mama ${ }^{1}$. A paciente em questão apresentou, posteriormente, esse tipo histológico.

Em relação ao perfil imuno-histoquímico, os tipos luminais A e B geralmente carregam um bom prognóstico e mostram uma resposta favorável para terapia endócrina. O luminal A apresenta melhor prognóstico que luminal $\mathrm{B}^{13}$.

Os cânceres de mama basal-símile correspondem ao grupo mais estudado. Esses grupos de tumores têm padrôes de expressão genética e características imuno-histoquímicas distintas. Apesar de não haver um consenso internacional no complemento preciso de marcadores que definem cânceres de mama basal-símile, a maioria dos autores considera a falta dos oncogenes ER, PR e HER-2, conhecidos como cânceres de mama triplo-negativos. Carcinomas de mama basal-símiles são caracterizados pela alta taxa mitótica e atividade proliferativa e geralmente apresentam uma grande área tumoral com distintas zonas celulares, compostas de material hialino, tecido isquêmico/ necrótico e colágeno ${ }^{13}$. 
Quando a pesquisa do oncogene HER-2 é positiva na imuno-histoquímica, os tumores são chamados de HER-2 e apresentam perfil biológico mais agressivo. Tal fato se apresenta em $10 \%$ dos tumores de mama ${ }^{14}$. São chamados tumores luminais híbridos aqueles em que há positividade para RP e/ou RE, associados a HER-2 positivo $^{14}$.

Analisando o perfil imuno-histoquímico do caso relatado, pôde-se perceber que a primeira lesão apresentada pela paciente foi classificada como triplo-negativo. Após quatro anos, quando a paciente apresentou lesão na mama contralateral, observou-se tumor MF, sendo que as duas lesôes divergiam entre si em relação ao seu padrão molecular, de forma que uma das lesôes apresentava padrão luminal A e a outra, padrão luminal híbrido.

Conforme estudo de Tolaney et al. ${ }^{9}$, a paciente foi submetida à associação de paclitaxel e trastzumabe adjuvante. Nessa pesquisa, observou-se taxa de sobrevida livre de doença invasiva em três anos de $98,7 \%{ }^{9}$. Além disso, conforme dados da metanálise do EBCTCG, quando completados dois anos de uso de tamoxifeno, a paciente realizará switch para inibidor de aromatase, até completar cinco anos de hormonioterapia.

\section{CONCLUSÃO}

Este estudo relata um caso de uma paciente que apresentou lesóes neoplásicas em ambas as mamas, em tempos distintos e com perfis histológicos e imuno-histoquímicos diferentes. Dessa forma, destacam-se a raridade do caso e a relevância da terapia dirigida a alvos específicos, uma vez que a paciente apresentava lesóes com perfis moleculares distintos.

\section{CONTRIBUIÇÕES}

Todos os autores contribuíram igualmente na concepção e/ou no planejamento do estudo; na obtenção, análise e interpretação dos dados; assim como na redação e revisão crítica; e aprovaram a versão final a ser publicada.

\section{AGRADECIMENTOS}

À Dra. Aline da Silva Freitas, médica oncologista do Serviço de Oncologia da Santa Casa de Misericórdia de Araraquara, SP, que contribuiu intelectualmente e na organização de informaçóes quanto ao plano terapêutico em realização pela paciente.

\section{DECLARAÇÃO DE CONFLITO DE INTERESSES}

Nada a declarar.

\section{FONTES DE FINANCIAMENTO}

Não há.

\section{REFERÊNCIAS}

1. Instituto Nacional do Câncer José de Alencar Gomes da Silva. A situação do câncer de mama no Brasil: síntese de dados dos sistemas de informação [Internet]. Rio de Janeiro: INCA; 2019 [acesso 2020 ago 7]. Disponível em: https://www.inca.gov.br/sites/ufu.sti. inca.local/files/media/document/a_situacao_ca_mama_ brasil_2019.pdf

2. Instituto Nacional de Câncer José de Alencar Gomes da Silva. Estimativa 2020: incidência de câncer no Brasil. Rio de Janeiro: INCA; 2019 [acesso 2020 ago 7]. Disponível em: https://www.inca.gov.br/sites/ufu. sti.inca.local/files//media/document//estimativa-2020incidencia-de-cancer-no-brasil.pdf

3. SIM: Sistema de Informação sobre Mortalidade [Internet]. Brasília, DF: Ministério da Saúde. [2017] [acesso 2020 ago 7]. Disponível em: http://tabnet. datasus.gov.br/cgi/tabcgi.exe?sim/cnv/obt10uf.def

4. Tot T, Pekár G, Hofmeyer S, et al. Molecular phenotypes of unifocal, multifocal, and diffuse invasive breast carcinomas. Patholog Res Int. 2011;2011:480960. doi: http://doi.org/10.4061/2011/480960

5. Jürgensen CM, Chacón CR, Baeza RC, et al. Cánceres de mama multifocales-multicéntricos: ¿Son realmente de peor pronóstico? Rev Chil Cir. 2009;61(2):125-30. doi: http://doi.org/10.4067/S0718-40262009000200004

6. El-Sheredy HG, El-Benhawy SA, Matrawy K, et al. Multifocal/multicentric versus unifocal breast câncer: what is the difference? Middle East J Cancer [Internet]. 2016 [cited 2020 Aug 7];7(2):69-78. Available from: http://mejc.sums.ac.ir/article_42031_5c6d0f372c0b8f 110289f936af88e83b.pdf

7. Navale P, Bleiweiss IJ, Jaffer S, et al. Evaluation of biomarkers in multiple ipsilateral synchronous invasive breast carcinomas. Arch Pathol Lab Med. 2019;143(2):190-6. doi: http://doi.org/10.5858/ arpa.2017-0494-OA.

8. Mosbah R, Rouzier R, Guinebretière JM, et al. Pathological characteristics of both tumors in bifocal and bicentric breast cancer. Anticancer Res. 2015 [cited 2020 Aug 7];35(9):5111-6. Available from: http:// ar.iiarjournals.org/content/35/9/5111.long

9. Tolaney SM, Barry WT, Dang CT, et al. Adjuvant paclitaxel and trastuzumab for node-negative, HER2positive breast câncer. N Engl J Med. 2015;372(2):13441. doi: http://doi.org/10.1056/NEJMoa1406281

10. Early Breast Cancer Trialists' Collaborative Group (EBCTCG). Aromatase inhibitors versus tamoxifen in early breast câncer: patient-level meta-analysis of the 
randomised trials. Lancet. 2015;386(10001):1341-52. doi: http://doi.org/10.1016/S0140-6736(15)61074-1

11. El mazghi A, Bouhafa T, Loukili K, et al. [Intracystic papillary carcinoma of the breast: report of three cases]. Pan Afr Med J. 2014;18:207. doi: http://doi. org/10.11604/pamj.2014.18.207.4519 French

12. Ingle SB, Murdeshwar HG, Siddiqui S. Papillary carcinoma of breast: minireview. World J Clin Cases. 2016;4(1):20-4. doi: http://doi.org/10.12998/wjcc. v4.i1.20

13. Masood S. Breast cancer subtypes: morphologic and biologic characterization. Womens Health (Lond). 2016;12(1):103-19. doi: http://doi.org/10.2217/ whe.15.99

14. Carmo PO, Leite ICG, Guerra MR. Sobrevida de mulheres com câncer de mama subtipo luminal assistidas em Juiz de Fora, MG. Rev Bras Mastologia [Internet]. 2016 [acesso 2020 mar 12];26(3):118-25. Disponível em: https://www.mastology.org/wp-content/ uploads/2016/06/MAS_v26n3_118-125.pdf 